\title{
Avaliação de Impactos Ambientais da duplicação da BR 101 RJ/Norte, trecho compreendido entre o KM 144,2 e 190,3
}

\author{
Marina Morim Gomes ${ }^{\prime}$; Flávia Constantino da Vitória²; Elenice Rachid da Silva \\ Josimar Ribeiro de Almeida ${ }^{l}$ \\ \llavia.c.vitoria@gmail.com
}

1. Universidade do Estado do Rio de Janeiro, Rio de Janeiro, Brasil 2. Universidade Federal Fluminense, Rio de Janeiro, Brasil 3. Universidade Federal do Rio de Janeiro, Rio de Janeiro, Brasil.

Histórico do Artigo:

Recebido em: 11 de julho de $2018 \quad$ Aceito em: 23 de outubro de $2018 \quad$ Publicado em: 30 de abril de 2019

Resumo: A BR 101 é uma rodovia de grande importância para o país e vêm sofrendo obras de duplicação para diminuir o número de congestionamentos e acidentes ao longo de sua extensão. 0 trecho compreendido entre o km 190,3 ao km 144,2 transpassa uma Unidade de Conservação (UC) e uma Área de Proteção Ambiental (APA), áreas onde a biodiversidade deve ser preservada, tendo seu amparo legal através de Lei 6938/81, Política Nacional do Meio Ambiente. Foram identificados onze impactos ambientais do meio biótico causados por este empreendimento e avaliados através de uma matriz de interação. 0s impactos foram negativos e revelam uma situação preocupante devido aos danos permanentes causados ao ecossistema adjacente, que podem afetar as populações da fauna e da flora.

Palavras-chave: Duplicação de rodovia, Impactos ambientais, Fragmentação de habitat, Matriz de interação.

\section{Evaluation of Environmental Impacts of doubling of BR 101 RJ/North stretch between KM 144.2 to 190.3}

\begin{abstract}
The BR 101 is a very important road for the country and have suffered duplication works to decrease the number of traffic jams and accidents along its length. The stretch between $\mathrm{km} 190.3$ to $\mathrm{km} 144.2$ pierces a Conservation Unit (UC) and an Environmental Protection Area (APA), areas where biodiversity must be preserved, and its legal support by Law 6938/8, National Environmental Policy. Eleven environmental impacts were identified caused by this project and evaluated through a matrix of interaction. The impacts were negative and reveal a worrying situation because of permanent damage to the ecosystem adjacent, which can affect the populations of fauna and flora.
\end{abstract}

Keywords: Road duplication, Environmental impacts, Habitat fragmentation, Interaction matrix.

\section{Évaluation des Impacts Environnementaux de la duplication de la BR $101 \mathrm{RJ} /$ norte comprenant les KM 144.2 et 190.3}

Resumen: La BR 101 es una carretera de gran importancia para el país y vienen sufriendo obras de duplicación para disminuir el número de congestiones y accidentes a lo largo de su extensión. El tramo comprendido entre el km 190,3 al km 144,2 traspasa una Unidad de Conservación (UC) y un Área de Protección Ambiental (APA), áreas donde la biodiversidad debe ser preservada, teniendo su amparo legal a través de Ley 6938/81, Política Nacional del Medio Ambiente. Se identificaron once impactos ambientales del medio biótico causados por este emprendimiento y evaluados a través de una matriz de interacción. Los impactos fueron negativos y revelaron una situación preocupante debido a los daños permanentes causados al ecosistema adyacente, que pueden afectar a las poblaciones de la fauna y la flora.

Palabras clave: Duplicación de carreteras, Impactos ambientales, Fragmentación de hábitat, Matriz de interacción. 
Avaliação de Impactos Ambientais da duplicação da BR 101 RJ/Norte, trecho compreendido entre o KM 144,2 e 190,3

\section{INTRODUÇÃO}

As atividades humanas ocasionam impactos ambientais quando afetam positivamente ou negativamente o meio ambiente ou alguns de seus elementos. Deste modo, a construção ou ampliação de uma infraestrutura rodoviária implicará na geração de significativos impactos. No Brasil, somente a partir da década de 80 é que efetivamente começou uma maior preocupação com as questões ambientais, devido às pressões exercidas pelos organismos multilaterais de financiamento para grandes projetos, pois nesta época o meio ambiente era visto como um entrave ao crescimento (SILVA \& SILVA, 2013).

Os Países Latino - americanos signatários do Tratado de Assunção de1991 e fundadores do MERCOSUL (Brasil, Argentina, Paraguai e Uruguai) contam com recomendações do GMCGrupo de Mercados Comuns, onde dentre algumas recomendações de cunho ambiental há o Acordo-Quadro sobre Meio Ambiente do MERCOSUL, a recomendação MERCOSUL/CMC/DEC. N ${ }^{\circ}$ 02/01, onde em seu capítulo II, Artigo $4^{0}$ descreve que o presente Acordo tem como objetivo o desenvolvimento sustentável e a proteção do meio ambiente mediante a articulação entre as dimensões econômicas, sociais e ambientais, contribuindo para uma melhor qualidade do meio ambiente e de vida das populações.

A política ambiental dos Estados Unidos é definida pelo National Environmental Policy Act - NEPA, tendo sido promulgada em $1^{0}$ de janeiro de 1970, e expõe princípios que devem ser seguidos pela Administração quando da implementação de projetos com relativo impacto ambiental, segundo Magrini (1989 apud R0CHA et al. 2005), a NEPA é considerada o principal marco da conscientização ambiental, sendo uma resposta às pressões crescentes da sociedade organizada para que os aspectos ambientais passassem a ser considerados na tomada de decisão sobre a implantação de projetos capazes de causar significativa degradação ambiental.

Diante da diversificação de impactos associados desde a fase de construção a implantação das rodovias, o meio ambiente é parte vulnerável nesse contexto, segundo Fogliatti et al (2004), os estudos ambientais desenvolvidos para estes empreendimentos devem caracterizar para cada fase as principais atividades impactantes e o meio impactado, com a finalidade de elaborar medidas de preservação, mitigadoras e até mesmo compensatórias, a fim de garantir que a intervenção humana na natureza seja a menor possível. 
As rodovias são importantes para a melhoria da qualidade de vida e tráfego entre cidades vizinhas, promovendo desenvolvimento socioeconômico (MAGALHÃES et al., 2011). Por ser um modal de transporte com menores custos fixos e flexibilidade em relação às origens e destino de cargas, apresentam vantagens significativas se comparadas aos demais modais.

A construção e manutenção de uma rodovia geram diversos impactos ambientais, que podem atingir o meio antrópico, físico e biótico (TROMBULAK \& FRISSEL, 1999; MAGALHÃES et al., 2011). A Resolução Conama $\mathrm{n}^{0}$ 001, de 23 de janeiro de 1986, em seu artigo $1^{0}$, considera impacto ambiental qualquer alteração das propriedades físicas, químicas e biológicas do meio ambiente, causada por qualquer forma de matéria ou energia resultante das atividades humanas que, direta ou indiretamente, afetam: a saúde, a segurança e o bem-estar da população; as atividades sociais e econômicas; a biota; as condições estéticas e sanitárias do meio ambiente; a qualidade dos recursos ambientais.

Todo empreendimento no modal rodoviário geram intervenções ambientais na área de influência direta e alteração ambiental nas áreas de influência indireta. Dentre os impactos ambientais provocados por projetos de transporte, estão: poluição do ar, ruído, intrusão visual, efeitos sobre o solo, efeitos sobre águas superficiais e subterrâneas, impacto sobre biota e segregação de comunidades. Para a mensuração dos impactos é necessário considerar alguns aspectos, sendo alguns deles: aspectos geológicos, hidrológicos, geomorfológicos, climáticos, ecossistemas, volume de desmatamento, interferência na infraestrutura existente, unidades de conservação ambiental, sítios arqueológicos, espécies de fauna e flora ameaçados e outros aspectos relevantes.

Embora seja possível predizer impactos que ocorrem na construção e manutenção de rodovias, é importante que cada caso seja estudado particularmente.

A BR 101 é uma rodovia federal, que cruza doze estados nas regiões Sul, Sudeste e Nordeste e é oficialmente denominada Rodovia Governador Mário Covas. Foi construída entre os anos 60 e 70, com duas pistas de sentidos contrários, e vêm passando por um processo de duplicação ao longo de diversos trechos da sua extensão, na tentativa de diminuir o número de acidentes e engarrafamentos (AUTOPISTA FLUMINENSE, 2014). 0 trecho compreendido entre 0 km 190,3 e o km 144,2, localizado no Estado do Rio de Janeiro, transpõem duas unidades de conservação, a Reserva Biológica União (REBIO UNIÃo) e Área de Proteção Ambiental (APA) da Bacia do Rio São João, exigindo uma avaliação cuidadosa dos impactos ambientais causados pela duplicação. 
Avaliação de Impactos Ambientais da duplicação da BR 101 RJ/Norte, trecho compreendido entre o KM 144,2 e 190,3

A partir do Estudo de Impactos Ambientais (EIA) realizado pela empresa de concessão rodoviária Autopista Fluminense (2014), em cumprimento de condicionante exigida para obtenção da Licença Prévia do empreendimento, o presente estudo pretende avaliar os impactos ambientais do meio biótico, fauna e flora, causados pela duplicação e se os mesmos são capazes de afetar drasticamente a dinâmica das Unidades de Conservação, a REBIO UNIÃo e APA da Bacia do Rio São João, inseridas dentro do trecho rodoviário objeto desse estudo. Assim, visando identificar os impactos relacionados com a fauna e a flora causados pela duplicação da rodovia BR 101 no trecho analisado, promover a avaliação da importância de cada impacto através da atribuição de valores categóricos aos mesmos e discutir cada impacto em particular e avaliação dos efeitos e consequências no empreendimento específico estudado.

\section{MATERIAL E MÉTODOS}

0 empreendimento, objeto deste estudo, é a rodovia BR 101 RJ/NORTE no trecho em duplicação compreendido entre o km 144,2 (N: 7.512.444 e E: 183.966) ao km 190,3 (N: 7.541.008 e E: 216.037), que percorre quatro municípios, sendo eles: Rio das Ostras, Macaé, Conceição de Macabu e Casimiro de Abreu. A área de estudo em questão transpassa a Reserva Ecológica União e a Área de Preservação Ambiental da Bacia do Rio São João e Áreas de Preservação Permanente.

Para avaliar os impactos ambientais, foi utilizado o Estudo de Impactos Ambientais (EIA) realizado pela Autopista Fluminense, disponibilizado publicamente pelo IBAMA (2013) em sua página eletrônica (site). Também foi consultada a base de dados do Google Scholar, utilizando as palavras-chaves "impactos ambientais", "rodovia", "enviromental impacts" e "road".

Com os dados obtidos, foi concretizada uma metodologia de Avaliação de Impactos Ambientais (AIA), a matriz de interação, levando em consideração apenas os aspectos do meio biótico, para melhor detalhamento do mesmo e realizada segundo Cremonez et al. (2014). Foram identificados onze impactos diferentes e utilizados sete critérios para classificar cada impacto: Natureza, Influência, Dimensão, Prazo, Ciclicidade e Reversibilidade e Importância.

A partir do conhecimento dos impactos ambientais causados durante as obras de duplicação da rodovia é possível mensurar seus efeitos na REBIO UNIÃo e APA da Bacia do Rio 
São João, a potencialidade desses impactos em alterar a dinâmica existente nas reservas ambientais e se serão negativos para a fauna e flora.

\section{RESULTADOS E DISCUSSÃ0}

Os impactos identificados foram: pisoteamento de flora e invertebrados, afugentamento da fauna e interferência de ruídos e vibrações, aumento do efeito de borda, aumento do nível de fragmentação do habitat, alteração da dinâmica das águas superficiais e profundas, redução da cobertura vegetal, intervenção em áreas de preservação e unidades de conservação, risco de supressão de espécies ameaçadas de extinção, alteração no nível de risco de ocorrência de incêndios florestais, alteração na ecologia da fauna aquática e atropelamento de fauna. Todos os impactos relacionados são negativos para o meio biótico, variando em natureza, influência, dimensão, prazo, ciclicidade e reversibilidade.

Tabela 1: Matriz de interações das ações realizadas durante o empreendimento x Impactos associados (X: presença).

\begin{tabular}{|c|c|c|c|c|c|c|c|c|c|c|c|c|}
\hline Ações & $\begin{array}{l}\frac{\pi}{0} \\
\frac{0}{0} \\
\frac{\pi}{0} \\
\frac{0}{0} \\
\frac{\pi}{0} \\
: 0 \\
=0\end{array}$ & 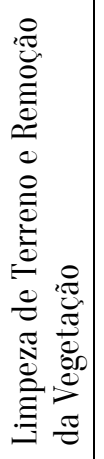 & 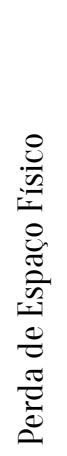 & 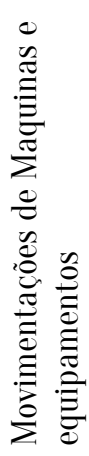 & 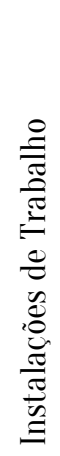 & 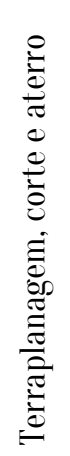 & 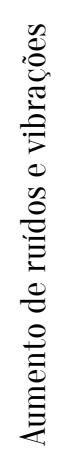 & $\begin{array}{l}0 \\
0 \\
0 \\
0 \\
0 \\
0 \\
0 \\
0 \\
0 \\
0 \\
0 \\
0 \\
0 \\
0 \\
0 \\
0 \\
0 \\
0 \\
0\end{array}$ & 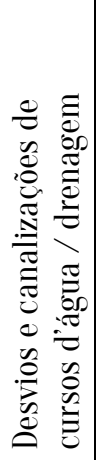 & 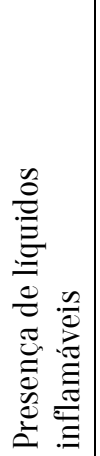 & 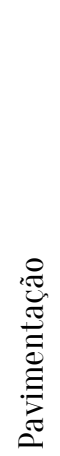 & 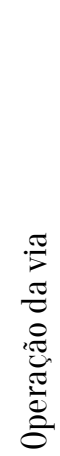 \\
\hline $\begin{array}{c}\text { Pisoteamento } \\
\text { de flora e } \\
\text { invertebrados }\end{array}$ & X & X & & & & & & & & & & \\
\hline $\begin{array}{c}\text { Afugentamento } \\
\text { da fauna e } \\
\text { interferência } \\
\text { de ruídos e } \\
\text { vibrações } \\
\end{array}$ & & & & & & & $\mathrm{X}$ & & & & & \\
\hline $\begin{array}{l}\text { Aumento do } \\
\text { efeito de borda }\end{array}$ & & X & X & & & & & & & & & \\
\hline $\begin{array}{l}\text { Aumento do } \\
\text { nível de } \\
\text { fragmentação } \\
\text { do habitat }\end{array}$ & & X & X & & & & & & & & & \\
\hline $\begin{array}{c}\text { Alteração da } \\
\text { Dinâmica das } \\
\text { Águas } \\
\text { Superficiais e } \\
\text { Profundas }\end{array}$ & & $\mathrm{X}$ & & X & $\mathrm{X}$ & X & & & & & $\mathrm{X}$ & \\
\hline Redução da & & $\mathrm{X}$ & & & $\mathrm{X}$ & & & $\mathrm{X}$ & & & & \\
\hline
\end{tabular}


Avaliação de Impactos Ambientais da duplicação da BR 101 RJ/Norte, trecho compreendido entre o KM 144,2 e 190,3

\begin{tabular}{|c|c|c|c|c|c|c|}
\hline $\begin{array}{c}\text { cobertura } \\
\text { vegetal }\end{array}$ & & & & & & \\
\hline $\begin{array}{c}\text { Intervenção em } \\
\text { Áreas de } \\
\text { Preservação e } \\
\text { Unidades de } \\
\text { Conservação }\end{array}$ & X & X & $\mathrm{X}$ & $\mathrm{X}$ & & \\
\hline $\begin{array}{c}\text { Risco de } \\
\text { supressão de } \\
\text { espécies } \\
\text { ameaçadas de } \\
\text { extinção }\end{array}$ & $\mathrm{X}$ & & & & & \\
\hline $\begin{array}{l}\text { Alteração no } \\
\text { nível de risco } \\
\text { de ocorrência } \\
\text { de incêndios } \\
\text { florestais }\end{array}$ & & & & & $\mathrm{X}$ & \\
\hline $\begin{array}{c}\text { Alteração na } \\
\text { ecologia da } \\
\text { fauna aquática }\end{array}$ & & & $\mathrm{X}$ & $X$ & & \\
\hline $\begin{array}{c}\text { Atropelamento } \\
\text { de fauna }\end{array}$ & & & & & & X \\
\hline
\end{tabular}

Fonte: Dados secundários utilizados foram obtidos através do Estudo de Impactos Ambientais (EIA) realizado pela Concessionária Autopista Fluminense.

Ao observar a Tabela 1, foi possível perceber que algumas ações causaram diversos impactos enquanto outras causaram apenas um. A ação que mais se destacou na matriz foi a limpeza de terreno e remoção da vegetação que causou cinco impactos diferentes, enquanto outros causaram apenas um impacto. 0 impacto causado por mais ações foi a Alteração da dinâmica das Águas Superficiais e Profundas, seguido da Intervenção em Áreas de Preservação e Unidades de Conservação, porém não existe relação entre o número de impactos causado por uma ação e a importância dos impactos.

Tabela 2: Matriz de Interações de Avaliação dos Impactos Ambientais identificados no meio biótico.

\begin{tabular}{|c|c|c|c|c|c|c|c|c|c|c|}
\hline MATRIZ DE INTERAÇÕES & \multicolumn{3}{|c|}{ FASES } & \multicolumn{7}{|c|}{ CRITÉRIOS } \\
\hline IMPACTOS & Planeja & Implan & Opera & Nat & $\operatorname{Inf}$ & Dim & Pra & $\mathrm{Cic}$ & Rev & $\operatorname{Imp}$ \\
\hline Pisoteamento de flora e invertebrados & $\mathrm{X}$ & - & - & $\mathrm{N}$ & $\mathrm{D}$ & $\mathrm{L}$ & $\mathrm{C}$ & $\mathrm{T}$ & I & B \\
\hline $\begin{array}{l}\text { Afugentamento da fauna e interferência } \\
\text { de ruídos e vibrações }\end{array}$ & - & X & X & $\mathrm{N}$ & D & $\mathrm{L}$ & M & $\mathrm{T}$ & $\mathrm{R}$ & A \\
\hline Aumento do efeito de borda & - & $\mathrm{X}$ & $\mathrm{X}$ & $\mathrm{N}$ & $\mathrm{I}$ & $\mathrm{R}$ & $\mathrm{L}$ & $\mathrm{P}$ & $\mathrm{I}$ & M \\
\hline $\begin{array}{c}\text { Aumento do nível de fragmentação do } \\
\text { habitat }\end{array}$ & - & X & X & $\mathrm{N}$ & D & $\mathrm{L}$ & $\mathrm{L}$ & $\mathrm{P}$ & I & A \\
\hline Redução da cobertura vegetal & - & 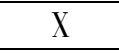 & & $\mathrm{N}$ & $\mathrm{D}$ & $\mathrm{L}$ & M & $\mathrm{T}$ & I & M \\
\hline $\begin{array}{c}\text { Intervenção em Áreas de Preservação e } \\
\text { Unidades de Conservação }\end{array}$ & - & X & X & $\mathrm{N}$ & D & $\mathrm{L}$ & M & $\mathrm{T}$ & I & A \\
\hline $\begin{array}{l}\text { Risco de supressão de espécies } \\
\text { ameaçadas de extinção }\end{array}$ & - & X & X & $\mathrm{N}$ & D & $\mathrm{L}$ & $\mathrm{C}$ & $\mathrm{T}$ & $\mathrm{R}$ & A \\
\hline
\end{tabular}




\begin{tabular}{|c|c|c|c|c|c|c|c|c|c|c|}
\hline $\begin{array}{c}\text { Alteração no nível de risco de ocorrência } \\
\text { de incêndios florestais }\end{array}$ & - & $\mathrm{X}$ & - & $\mathrm{N}$ & $\mathrm{D}$ & $\mathrm{L}$ & $\mathrm{M}$ & $\mathrm{T}$ & $\mathrm{R}$ & $\mathrm{B}$ \\
\hline Alteração na ecologia da fauna aquática & - & $\mathrm{X}$ & $\mathrm{X}$ & $\mathrm{N}$ & $\mathrm{I}$ & $\mathrm{R}$ & $\mathrm{L}$ & $\mathrm{P}$ & $\mathrm{I}$ & $\mathrm{M}$ \\
\hline $\begin{array}{c}\text { Alteração da Dinâmica das Águas } \\
\text { Superficiais e Profundas }\end{array}$ & - & $\mathrm{X}$ & $\mathrm{X}$ & $\mathrm{N}$ & $\mathrm{D}$ & $\mathrm{L}$ & $\mathrm{L}$ & $\mathrm{P}$ & $\mathrm{R}$ & $\mathrm{M}$ \\
\hline Atropelamento de fauna & - & - & $\mathrm{X}$ & $\mathrm{N}$ & $\mathrm{D}$ & $\mathrm{L}$ & $\mathrm{L}$ & $\mathrm{P}$ & $\mathrm{I}$ & $\mathrm{A}$ \\
\hline
\end{tabular}

Fonte: Dados secundários utilizados foram obtidos através do Estudo de Impactos Ambientais (EIA) realizado pela Concessionária Autopista Fluminense.

Legenda: Planeja - Planejamento, Implan - Implantação, Opera - Operação (X: presença/-:ausência);Nat- Natureza (N: Negativa/P: Positiva); Inf - Influência (D:Direta/I:Indireta); Dim - Dimensão (L: local/R: Regional); Pra - Prazo (C: Curto/M: Médio/L: Longo); Cic - Ciclicidade (T: Temporário/P: Permanente); Rev - Reversibilidade (R: Reversível/I: Irreversível); Imp - Importância (B: Baixa/M: Média/A: Alta).

Seguindo a Resolução CONAMA 01/86, a segunda matriz foi elaborada levando em conta aspectos descritivos para que fosse possível determinar o grau de importância de cada impacto. É importante ressaltar que os impactos identificados também ocorreram quando da construção deste trecho da BR 101, porém possuem importâncias diferentes nos dois empreendimentos. 0 aumento do efeito de borda, por exemplo, foi mais impactante quando da construção da estrada, já que dividiu áreas de Mata Atlântica em dois fragmentos.

\section{Pisoteamento de flora e fauna}

Os profissionais da área da biologia têm consciência que a presença dos mesmos nas áreas de pesquisa já é um impacto por si só. Durante o processo de amostragem, vegetais e pequenos invertebrados, principalmente artrópodes, são pisoteados e, muitas vezes, mortos. Este impacto possui pouca importância se comparado com todos os outros causados pela construção e/ou duplicação de uma rodovia, porém não deveria ser ignorado.

\section{Afugentamento de fauna e interferência de ruídos e vibrações}

A resposta ao barulho do tráfego de veículos é um dos motivos pelo qual os animais evitam a estrada (SHIER et al., 2012). Diversos répteis, muitas aves e mamíferos utilizam o som para comunicação, rituais de reprodução, para encontrar a presa (forragear) e são afetados pelo aumento de ruído (HALFWERK, 2011). Diversos efeitos negativos podem ser causados pelo aumento de ruído: problemas na comunicação, aumento dos hormônios de estresse, espanto da presa (SHIER et al., 2012). Os morcegos são particularmente afetados por este impacto, já que utilizam a ecolocação para se locomover e forragear e alguns utilizam também o som emitido pelas presas para encontra-las e tendem a evitar os locais com alto nível de ruído (HALFWERK, 2011). 
Avaliação de Impactos Ambientais da duplicação da BR 101 RJ/Norte, trecho compreendido entre o KM 144,2 e 190,3

A duplicação da rodovia causará o aumento dos ruídos e vibrações durante as obras, devido a operação de máquinas e possibilitará o aumento dos mesmos durante a fase de operação, já que será possível passar mais veículos no mesmo espaço de tempo.

\section{Aumento do efeito de borda}

Espaços limpos lineares, abertos para a construção de rodovias, causam efeitos importantes em diversas espécies, aumentam o risco de bioinvasão, aumentam a circulação de humanos e facilitam a caça (LAURENCE et al., 2011). Existe uma quebra no microclima do fragmento na borda de uma estrada, onde a temperatura, umidade, nível de evaporação, influência do vento são diferentes do que aqueles dentro do ecossistema (PRIMAK, 2012). Desta forma, apenas indivíduos capazes de sobreviver nesse ambiente considerado estressante para aqueles organismos que não estão acostumados vão habitar a borda (BARBAR0 et al, 2012).

0 efeito de borda foi um impacto muito mais expressivo quando da abertura deste trecho da BR 101, já que houve a divisão do fragmento. Com a duplicação, o efeito de borda já existente se desloca na direção do fragmento, já que uma área da borda do fragmento foi perdida. Esse fenômeno pode ser perigoso para fragmentos pequenos, pois o efeito de borda tende a ser maior em fragmentos menores (DIDHAM et al, 2012).

\section{Aumento do nível de fragmentação do habitat}

As rodovias funcionam como uma barreira ou filtro para o movimento de diversas espécies (SHIER et al., 2012). A construção de estradas faz com que alguns grupos de animais evitem a proximidade com as mesmas, o que diminui a acessibilidade a outros recursos e aumenta o nível de fragmentação do habitat (BENÍTEZ-LÓPEZ et al., 2010). 0 efeito de barreira das rodovias tende a criar metapopulações, o que por sua vez coloca em risco a existência de populações em pequenos fragmentos já que a própria rodovia diminui ou impede a recolonização dos mesmos (SHIER et al, 2012). 0 aumento do nível de fragmentação diminui 0 fluxo gênico entre os indivíduos, causando diversos problemas genéticos e tornando a população mais susceptível a eventos de deriva genética (PRIMAK, 2012, J0HANSSON et al., 2013). Embora esse impacto já existia com a construção da rodovia, a duplicação da mesma causará a diminuição de área dos fragmentos, aumentando o nível de fragmentação.

\section{Redução da cobertura vegetal}


Para limpar o terreno, construir instalações de trabalho e trocar o solo, é necessário que a cobertura vegetal seja removida. A redução da cobertura vegetal tem diversos impactos, desde locais a regionais, como o aumento do escoamento superficial (FERNANDES et al., 2013), a diminuição da evapo-transpiração, a capacidade de reter água, além de afetar os organismos que utilizam a vegetação como esconderijo ou local para nidação (SHIER 2012).

\section{Alteração na Dinâmica das Águas Superficiais}

0 aumento da área pavimentada causa um importante impacto: 0 aumento do escoamento superficial. Esse aumento está associado com maior taxa de erosão, alteração da morfologia dos canais e pode causar alagamento do solo, o que impede a devida oxigenação das raízes e causa mortandade de vegetais (SHIER et al., 2012). É relevante destacar que não apenas a impermeabilização do solo (pavimentação) causa 0 aumento do escoamento superficial, mas também a remoção da vegetação, já que a mesma perde sua capacidade de reter água.

\section{Intervenção em Áreas de Preservação e Unidades de Conservação}

0 trecho compreendido entre o km 190 ao 144 da BR 101 cruza a ReBio União e a APA da Bacia do Rio São João. Desta forma, haverá supressão de vegetação para abertura de espaço para a pista. Este impacto possui uma alta importância (Tabela 2) e está diretamente relacionado com outros impactos, como a redução da cobertura vegetal. Porém nos trechos que a BR transpassa a Unidade de Conservação e a APA, todos os impactos relacionados na matriz possuem uma importância maior, já que é uma área onde segundo a Lei 6938/81, Política Nacional do Meio Ambiente, o objetivo é a proteção da biodiversidade.

\section{Risco de supressão de espécies ameaçadas de extinção}

Durante as atividades relacionadas à limpeza do terreno, haverá necessidade de exemplares arbóreos da espécie Dalbergia nigra (Vell.) Alemão ex Benth., que consta na lista oficial das espécies da flora brasileira ameaçadas de extinção, devido a sua intenção exploração para fins comerciais (MATOS et al., 2015).

\section{Alteração no nível de risco de ocorrência de incêndios florestais}

A presença de produtos inflamáveis para alimentar os equipamentos durante as obras de duplicação aumenta a exposição e, consequentemente, o risco de incêndios. Nos 
Avaliação de Impactos Ambientais da duplicação da BR 101 RJ/Norte, trecho compreendido entre o KM 144,2 e 190,3

ecossistemas que não dependem das queimadas naturais periódicas, o fogo pode ser uma ameaça à biodiversidade, já que gera um nível de distúrbio alto e os organismos que não possuem adaptações para resistir as queimadas acabam morrendo (W00STER et al., 2012).

\section{Alteração na ecologia da fauna aquática}

0 aumento do escoamento superficial e a produção de sedimentos são os principais processos físicos causados por rodovias que impactam ecossistemas aquáticos (SHIER et al., 2012). Com a construção e operação da estrada, a taxa de sedimentos suspensos no ar aumenta e podem ser depositados em corpos d'água, mudando a morfologia dos mesmos através da sedimentação, matando organismos aquáticos e diminuindo a produtividade devido ao aumento de turbidez (GRIFT et al., 2013).

\section{Atropelamento de fauna}

Após o término das obras, a rodovia possuirá o dobro de sua espessura, o que aumentará espaço a ser atravessado por animais e, consequentemente, o risco da fauna ser atropelada por veículos circulantes. A mortalidade de animais por colisão com veículos atinge a maioria das espécies terrestres e tem efeitos perceptíveis na demografia das populações, podendo atuar como um fator de aumento de fragmentação de habitat, já que algumas espécies de movimento lento e/ou alta migração no trecho rodoviário acabam por isolar suas populações (GRIFT et al., 2013). Espécies que são atraídas pelas estradas, não evitam as estradas ou possuem movimento lento são as mais vulneráveis (RYTWINSKI \& FAHRIG, 2011). Anfíbios e Répteis têm o maior registro de atropelamentos (BENÍTEZ-LÓPEZ et al., 2010).

Por cruzar duas Unidades de Conservação, este é o Impacto de maior relevância causado pelo empreendimento, já que pode gerar o isolamento genético de algumas populações. Embora não possa ser evitado, o atropelamento de fauna pode ser mitigado, através de medidas como posicionamento de espelhos para que os animais retornem, avisos na rodovia para os motoristas sobre as áreas de risco e implementação de travessias de fauna (AUSTIN, 2007).

\section{Influência dos impactos em diferentes grupos}

Animais maiores são mais vulneráveis a estradas por possuírem maior mobilidade, taxas reprodutivas menores e ocorrerem em densidades menores do que pequenos animais. As estradas, portanto, podem causar o aumento das populações de pequenos animais, se estes 
forem presas de animais maiores, através do efeito top-down (BENÍTEZ-LÓPEZ et al., 2010). Segundo a metodologia utilizada, os répteis e os mamíferos são os grupos mais afetados pelo empreendimento estudado, já que sofrem influência de diversos impactos. Porém, não foi realizado levantamento da fauna de invertebrados e a existência de poucas pesquisas avaliando o efeito de estradas nesse grupo dificultou a avaliação dos impactos neste grupo.

\section{CONCLUSÃO}

Os impactos referentes ao meio biótico do empreendimento em estudo foram negativos, devido às consequências na ecologia e dinâmica dos ecossistemas aquáticos e terrestres causadas pela duplicação da via, revelando um quadro preocupante, principalmente levando em consideração a Unidade de Conservação e a Área de Proteção Ambiental. Os fatores estudados influenciam não apenas na área adjacente à rodovia, mas ao ecossistema circundante como um todo, pois interferem nas relações ecológicas entre os diversos grupos de organismos. 0s impactos analisados mostraram que a duplicação das rodovias intensifica os impactos já causados pela construção da mesma, principalmente devido à fragmentação do habitat que possui consequências irreversíveis, como a diminuição do número de indivíduos abaixo do valor mínimo viável de uma população (SHIER et al., 2012).

Porém, não foram analisados os impactos relacionados ao meio antrópico, que incluem impactos positivos, como a redução do número de acidentes na via e a geração de empregos. A empresa responsável pelo empreendimento propôs diversas atividades mitigatórias para os impactos relacionados no Estudos de Impactos Ambientais (EIA) que poderão ser eficientes. Para melhor compreender os impactos e a eficiência das atividades mitigatórias, outros estudos devem ser realizados, principalmente no âmbito de acompanhar as mudanças que ocorrerão durante após a implantação do projeto, para que haja uma maior compreensão dos impactos causados.

\section{REFERÊNCIAS BIBLIOGRÁFICAS}

AUTOPISTA FLUMINENSE S/A. Estudo de Impacto Ambiental Duplicação da Rodovia Mario Covas BR-101/RJ Trecho: kml44+200 ao $\mathrm{km}$ 190+300. Novembro de 2013. Disponível em: http://licenciamento.ibama.gov.br/Rodovias/. Acesso em: 04 jul 2015.

AUSTIN, M. Species distribution models and ecological theory: A critical assessment and some possible new approaches. Ecological modeling, Holanda, v.200, p.1-19, 2007. 
Avaliação de Impactos Ambientais da duplicação da BR 101 RJ/Norte, trecho compreendido entre o KM 144,2 e 190,3

BARBARO, L.; BROCKERHOFF, B.G.; VAN HALDER, I. Edge and área effects on avian assemblages and insectivory in fragmented native forests. Landscape Ecology, União Européia, v.27, p. 1451-1463, 2012.

BENÍTEZ-LÓPEZ, A.; ALKEMADE, R.; VERWEIJ, P.A. The Impact of Roads and Other Infrastructure on Mammal and Bird Population: A Meta-analysis. Biological Conservation, Holanda, v.143, p.1307-1316, 2010.

BRASIL. Lei $n^{0} 6.938$ de 31 de agosto de 1981. Dispõe sobre a Política Nacional do Meio Ambiente, seus fins e mecanismos de formulação e aplicação, e dá outras providências. Brasília, 31 de agosto de 1981.

BRASIL. Conselho Nacional de Meio Ambiente IBAMA. Resolução CONAMA 001, de 23 de janeiro de 1986. Estabelece as definições, as responsabilidades, os critérios básicos e as diretrizes gerais para uso e implementação da Avaliação de Impacto Ambiental como um dos instrumentos da Política Nacional do Meio Ambiente. Brasília, 23 de janeiro de 1986.

CREMONEZ, F.E.; et al. Avaliação de Impacto Ambiental: Metodologias aplicadas no Brasil. Revista Monografias Ambientais, Santa Maria, v.13, n.5, p.3821-3830, 2014.

DIDHAM, R.K.; KAPOS, V.; EWERS, R.M. Rethinking the conceptual foundations of habitat fragmentation research. Oikos, Rio de Janeiro, v.121, n.2, p. 161-170, 2012.

FERNANDES, R.P.; et al. Geração de escoamento superficial em uma microbacia com cobertura de cana-de-açúcar e floresta ripária. Rev. Ambient. Água, Taubaté, v.8, n. 3, p. 178-190, 2013.

F0GLIATTI, M.C.; FILIPPO, S.; GOUDARD, B. Avaliação de impactos ambientais: aplicação ao sistema de transportes. 1 Ed, Rio de Janeiro: Editora Interciência, 2004.

GOUDIE, A.S. The Human Impact of the Natural Enviroment: Past, Present and Future. 7ed., Wiley-Blackwell, 2013

GRIFT, E.A.; et al. Evaluating the effectiveness of road mitigation measures. Biodiversity and Conservation , Holanda, v.22, p.425-448, 2013.

HALFWERK, W.; et al. Negative Impacto f traffic noise on avian reproductive success. Journal of Applied Ecology, Reino Unido, v.48, p.210-219, 2011.

HAMMIT, W.E.; COLE, D.N.; MONZ, C.A. Wildland Recreation: Ecology and Management, 3. Ed, Wiley-Blackwell, EUA, 2015.

JOHANSSON, T.; et al. Environmental considerations from legislation and certification in managed forest stands: A review of their importance for biodiversity. Forest Ecology and Management, Holanda, v.303, n.lp. 98-112.

LAURANCE, W.F.; et.al. The fate of Amazonian Forest Fragments: a 32-year investigation. Biological Conservation, Holanda, v.144, p. 56-67, 2011.

MAGALHÃES, I.A.L; MARTINS, R.F.; SANTOS, R.A. Identificação dos Impactos Ambientais Relacionados à Pavimentação da Rodovia MG 307 no município de Grão Mogol - MG. Revista Verde, Moçoró, v.6, n.5 p.10-16, 2011.

MERCOSUL - página brasileira do MERCOSUL. Normativas. Disponível em http://www.mercosul.gov.br/normativa. Acesso em 07 jul 2018.

MATOS, A.C.B; BORGES, E.E.L.; SILVA, L.J. Fisiologia da germinação de sementes de Dalbergia nigra (Vell.) Allemão ex Benth. Sob diferentes temperaturas e tempos de exposição. Revista Árvore, Viçosa, v.39, n.l, 2015.

PRIMACK, R.B. A primer of conservation biology. 5 ed. Massachusetts, Sinauer Associates, 2012.

RYTWINSKI, T.; FAHRIG, L. Reproductive rate and body size predict road impacts on mammal abundance. Ecological Applications, Canadá, v.21, n.2, p. 589-600, 2011.

ROCHA, E. C.; CANT0, J.L.; PEREIRA, P.C. Avaliação de impactos ambientais nos países do MERCOSUL. Ambiente \& Sociedade - Vol. VIII n ${ }^{0} .2$ jul./dez. 2005. 
SHIER, D.M.; LEA, A.J.; OWEN, M.A. Beyond masking: Endangered Stephen's kangaroo rats respond to traffic noise with footdrumming. Biological Conservation, EUA, v.150, p. 53-58, 2012.

SILVA, M. P.; DA SILVA, D. S.. Avaliação de impactos ambientais em projeto rodoviário urbano: estudo de caso Americana/SP. Revista Ciência e Tecnologia, Piracicaba, v. 16, n. 28/29, ISSN 2236-6733, jan. 2014.

TROMBULAK, S.C.; FRISSEL, C.A. Review of Ecological Effects of Roads on Terrestrial and Aquatic Comunmunities. Conservation Biology, EUA, v.14, n.l, p.18-30, 1999.

WOOSTER, M.J.; PERRY, G.L.W.; ZOUMAS, A. Fire, drought and El Niño relationships on Borneo (Southeast Asia) in the pre-MODIS era (1980-2000). Biogeosciences, União Europeia, v.9, p. 317-340, 2012. 